\title{
Genetic and physiological characterization of rpoB mutations that activate antibiotic production in Streptomyces lividans
}

\author{
Caixia Lai, $†$ Jun Xu, Yuzuru Tozawa, $\ddagger$ Yoshiko Okamoto-Hosoya, \\ Xingsheng Yaot and Kozo Ochi
}

National Food Research Institute, 2-1-12 Kannondai, Tsukuba, Ibaraki 305-8642, Japan

\author{
Author for correspondence: Kozo Ochi. Tel: +8129838 8125. Fax: + 81298387996. \\ e-mail : kochi@affrc.go.jp
}

\begin{abstract}
Antibiotic production in Streptomyces lividans can be activated by introducing certain mutations (rif) into the rpoB gene that confer resistance to rifampicin. Working with the most typical (rif-17) mutant strain, KO-417, the rif-17 mutation was characterized. The rif-17 mutation was shown to be responsible for activating antibiotic production and for reducing the growth rate of strain KO-417, as demonstrated by gene-replacement experiments. Gene-expression analysis revealed that introduction of rif into $S$. lividans elevates expression of the pathway-specific regulatory gene actII-ORF4 to nearly the same level seen in Streptomyces coelicolor. The rif effect on antibiotic production was still evident in the genetic background of re/C, indicating that the rif mutation can provoke its effect without depending on ppGpp. Accompanying the restoration of antibiotic production, rif mutants also exhibited a lower rate of RNA synthesis compared to the parental strain when grown in a nutritionally rich medium, suggesting that the mutant RNA polymerases may behave like 'stringent' RNA polymerases. These results indicate that the rif mutation can alter the gene-expression pattern independent of ppGpp. The impaired growth of strain K0-417 (rif-17) was largely restored by introducing the second rif mutation (rif-18) just adjacent to the rif-17 position. Proteome analysis using two-dimensional PAGE revealed that the rif mutant strain KO-418 (rif-17 rif-18) displayed a temporal burst of expression especially of two enzymes, glutamine synthetase (type II) and oxidoreductase, during the late growth phase.
\end{abstract}

Keywords: RNA polymerase, stringent response

\section{INTRODUCTION}

Antibiotic production in Streptomyces spp. is believed to occur in a growth-phase-dependent manner (Martin \& Liras, 1989; Demain \& Fang, 1995; Bibb, 1996). The initiation of antibiotic biosynthesis usually starts at the transition between vegetative growth and morphological development (Chater, 1993). While the physiological signals and underlying regulation mechanisms that regulate antibiotic production remain to be fully eluci-

\footnotetext{
† Present address: Department of Pharmaceutics, Shenyang Pharmaceutical University, Shenyang 110015, China.

$\ddagger$ Present address: Mitsubishi Kagaku Institute of Life Sciences, Yokohama Research Center, 1000, Kamoshida-cho, Aoba-ku, Yokohama 227-8502, Japan.

Abbreviations: Act, actinorhodin; Red, undecylprodigiosin; Rif, rifampicin.
}

dated, most of the published data are consistent with the intrinsic role of growth rate (the cessation of growth) on the onset of secondary metabolism (Champness \& Chater, 1994). Streptomyces coelicolor A3(2), the most frequently studied species of Streptomyces, takes advantage of four kinds of antibiotics that it produces, including the pigmented antibiotics actinorhodin (Act) and undecylprodigiosin (Red). Streptomyces lividans is a close relative of $S$. coelicolor A3(2) and its merits include fast growth, good sporulation and almost no restriction of foreign DNA (Kieser et al., 2000). A large proportion of the $S$. lividans genome is identical to that of S. coelicolor A3(2), and the established genetic and physical maps of these two species are well-aligned to each other (Leblond et al., 1993). However, the Act and Red genes are normally poorly expressed in S. lividans. Why S. lividans can not produce Act, even though it carries the entire biosynthetic gene clusters required for 
Table 1. S. lividans strains used in this study

\begin{tabular}{|lll|}
\hline Strain & \multicolumn{1}{c|}{ Relevant genotype } & \multicolumn{1}{c|}{ Source/reference } \\
\hline 1326 & Prototrophic wild-type & D. A. Hopwood* ; Kieser et al. $(2000)$ \\
KO-417 & rif-17 & Rif-resistant isolate from 1326; Hu et al. (2002) \\
KO-418 & rif-17 rif-18 & Double Rif-resistant isolate from KO-417; this study \\
KO-419 & rif-17 rif-12 & Double Rif-resistant isolate from KO-417; this study \\
KO-420 & rif-positive & Spontaneous revertant isolate from KO-417; this study \\
KO-421 & relC & rplK mutant of 1326; this study \\
KO-422 & relC rif-1 & Rif-resistant isolate from KO-421; this study \\
KO-423 & relC rif-2 & Rif-resistant isolate from KO-421; this study \\
\hline
\end{tabular}

*Department of Genetics, John Innes Centre, Norwich Research Park, Colney, Norwich NR4 7UH, UK.

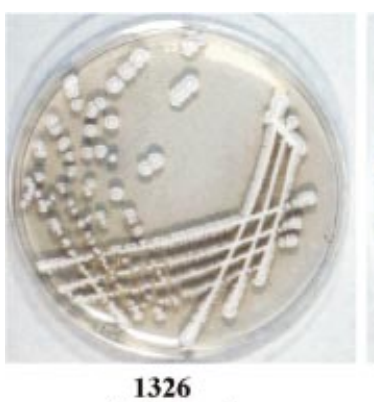

(wild-type)

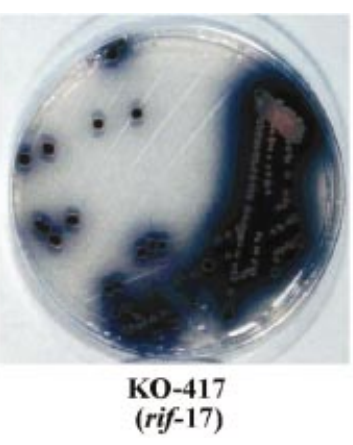

(rif-17)

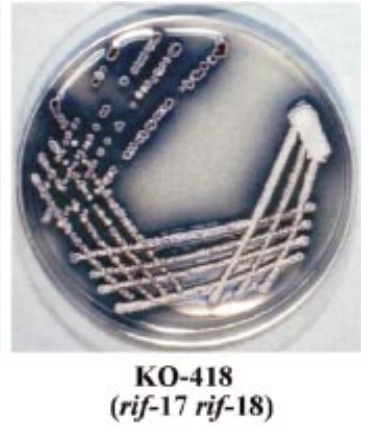

Fig. 1. Growth and Act production in the $S$. lividans wild-type and mutant strains. Spores were spread on R4 agar and then incubated at $30^{\circ} \mathrm{C}$ for 3 days. Blue colour represents the antibiotic Act. production of this antibiotic, is a point of interest. How to activate this silent antibiotic biosynthetic gene cluster can also give some clues about the regulation system for antibiotic production. We have previously reported that the introduction of the str mutation, which confers resistance to streptomycin, into $S$. lividans could activate Act production in this organism (Shima et al., 1996). Introducing the str mutation into other Streptomyces spp. was also effective in improving their antibiotic production (Hosoya et al., 1998). Moreover, the str mutation could also suppress the detrimental effect of relA and relC mutations on antibiotic production caused by the failure of strains to accumulate ppGpp (Ochi et al., 1997; Shima et al., 1996).

In a previous study (Hu et al., 2002), we reported that the introduction of certain Rif-resistance mutations (rif) into S. lividans can activate Act and Red production in this organism. These rif mutations were frequently found in the so-called rif domain within the $r p o B$ gene, which encodes the RNA polymerase $\beta$-subunit. In the present study, we have attempted to characterize these rif mutations by using both genetic and physiological approaches, including proteome analysis.

\section{METHODS}

Bacterial strains, plasmids and culture conditions. The $S$. lividans strains used in this study are listed in Table 1. Spontaneous rif mutants from S. lividans were obtained from colonies that grew within 7 days of spores being spread onto
GYM agar containing $100 \mu \mathrm{g}$ Rif $\mathrm{ml}^{-1}$. The mutants were used for subsequent study after single-colony isolation. GYM, R2YE and R4 media have been described previously (Kieser et al., 2000; Ochi, 1987; Shima et al., 1996). All cultivations were carried out at $30{ }^{\circ} \mathrm{C}$. Escherichia coli K-12 strains DH5 $\alpha$ and DM1 (dam $d c m ~ h s d R^{-} M^{+}$) (Invitrogen) were used for subcloning.

Screening of relC mutants. Screening of the relC mutants was carried out as described previously (Ochi et al., 1997). The $140 \mathrm{bp} \mathrm{rplK}$ fragments from the wild-type and thiostreptonresistant mutants of $S$. lividans, which developed after 7 days incubation on GYM agar medium containing $50 \mu \mathrm{g}$ thiostrepton $\mathrm{ml}^{-1}$, were amplified using colony PCR. Primer 1 (Forward, 5'-ATGCCTCCCAAGAAGAAGAA-3') and primer 2 (Reverse, 5'-GCGTTGTAGGCCTTGCAGAA-3') were designed from the $S$. coelicolor rplK gene sequence (DDBJ accession no. D83746; Shima et al., 1996). PCR and sequencing conditions were as described previously (Ochi et al., 1997).

Isolation and manipulation of DNA. Plasmid and total DNA were isolated from $S$. lividans as described by Kieser et al. (2000). Protoplast transformation was also done as described by Kieser et al. (2000). Southern analysis was performed using digoxigenin (DIG)-labelled probes made by random oligonucleotide priming (DIG DNA-labelling kit; Boehringer Mannheim). E. coli strains were grown and transformed using standard protocols (Sambrook et al., 1989).

Mutation analysis of rpoB. All primers used for checking chromosomal mutations in $r p o B$ were designed using sequence information obtained from S. coelicolor M145 (accession no. AL160431; Redenbach et al., 1996). The nucleotide sequence for the PCR fragment was determined by the dideoxynucleo- 


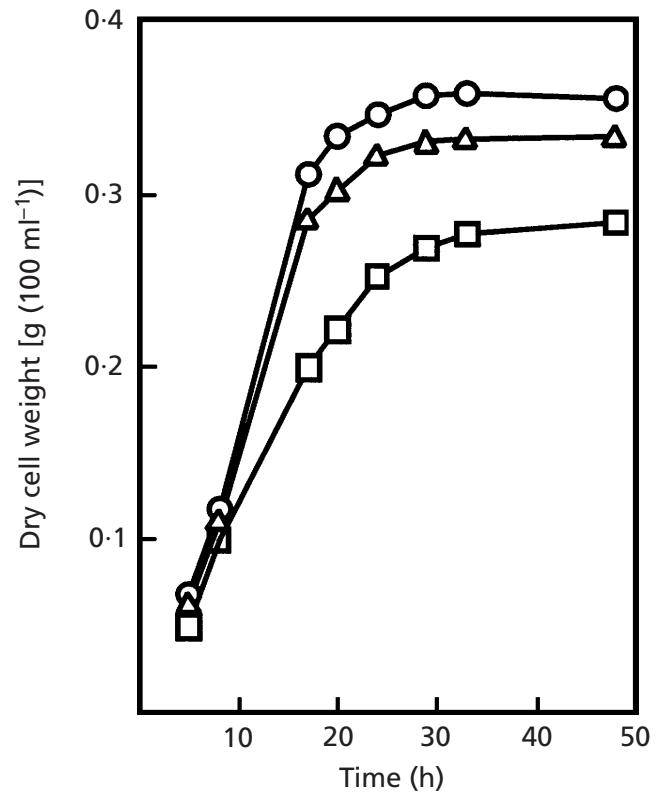

Fig. 2. Growth of $S$. lividans strains in GYM liquid medium. $\bigcirc$, Wild-type strain 1326; $\square$, KO-417 (rif-17); $\triangle$, KO-418 (rif-17 rif-18).

tide chain termination method using the BigDye Terminator Cycle Sequencing Kit (Perkin Elmer).

Gene-replacement analysis of rpoB. The procedure used for gene-replacement analysis is illustrated in Fig. 4. Total chromosomal DNA was prepared from the two rif mutants KO-417 (rif-17 Act-positive) and KO-418 (rif-17 rif-18 Actpositive). A $4354 \mathrm{bp} \mathrm{SacI-SacI} \mathrm{fragment} \mathrm{from} \mathrm{KO-417} \mathrm{and}$ $\mathrm{KO}-418$ was cloned into the SacI site of the multiple-cloning sites in a pBluescriptSK $(+)$ vector to generate pLC1 and pLC2, respectively. The insert includes the $r p o B$ coding region but lacks 15 aa residues at the carboxyl terminus. A $1 \cdot 1 \mathrm{~kb}$
EcoRI-EcoRI fragment containing the apramycin-resistance gene $(\operatorname{aac}(3) I V$; accession no. X99319) was cloned into both pLC1 and pLC2 in a step-wise manner, generating pLC3 and pLC4, respectively. Plasmids pLC3 and PLC4 were passaged through the methylation-deficient E. coli strain DM1 (dam $\mathrm{dcm}$ ) and introduced into $S$. lividans strains as described previously (Kieser et al., 2000). The R2YE plates were flooded with $1 \mathrm{ml}$ of an apramycin (Sigma) solution, to give a final concentration of $50 \mu \mathrm{g} \mathrm{ml}^{-1}$. Integration and looping-out of the plasmids by homologous recombination were confirmed by Southern hybridization.

Western blotting and RT-PCR analysis. Western analysis was carried out as described previously (Hu et al., 2002). RT-PCR was carried out by using the Thermoscript RT-PCR System Kit (Invitrogen).

Assay for ppGpp and determination of RNA synthesis. The intracellular ppGpp content was assayed as described by Ochi (1987) using HPLC analysis. RNA synthesis after Casamino acid deprivation or during growth in a Casamino acid medium was determined by measuring $\left[2-{ }^{14} \mathrm{C}\right]$ uracil incorporation into acid-precipitable material, as described previously (Ochi, 1990a).

Two-dimensional gel electrophoresis. Cells were collected from a GYM plate covered with a cellophane sheet. They were then disrupted by sonication three times for $30 \mathrm{~s}$ on ice, and centrifuged at $14000 \mathrm{~g}$ for $20 \mathrm{~min}$. The supernatants were used as protein extracts and $150 \mu \mathrm{g}$ total protein from each sample was applied to an Immobiline Dry Strip ( $\mathrm{pH} 4-7,18 \mathrm{~cm}$; Amersham Pharmacia) for isoelectric focusing using the Multiphor II Electrophoresis Unit (Amersham Pharmacia). An ExcelGel (XL SDS 12-14\% ; Amersham Pharmacia) was used for the second dimension SDS-PAGE.

Peptide-mass-fingerprinting analysis and $\mathbf{N}$-terminal-sequencing analysis. The gels were stained with Coomassie blue. Spots of interest were cut out and subjected to peptidemass-fingerprinting analysis and $\mathrm{N}$-terminal-sequencing analysis. Gel pieces were washed and dried under vacuum, before being digested with trypsin (Promega). After trypsin treatment, peptides were extracted with $25 \mu \mathrm{l}$ of $50 \%$

Table 2. Position of mutations in rpoB of S. lividans

\begin{tabular}{|c|c|c|c|c|c|}
\hline Strain & $\begin{array}{l}\text { Position of mutation } \\
\text { in } r p o B^{*}\end{array}$ & $\begin{array}{l}\text { Amino-acid } \\
\text { position }\end{array}$ & $\begin{array}{l}\text { Amino-acid } \\
\text { exchange }\end{array}$ & $\begin{array}{c}\text { Resistance to Rif } \\
\left(\mu \mathrm{g} \mathrm{ml}^{-1}\right) \dagger\end{array}$ & Act production $\neq$ \\
\hline 1326 & $-\mathbb{S}$ & & & 10 & - \\
\hline KO-417 & $1318(\mathrm{C} \rightarrow \mathrm{T})$ & 440 & Arg $\rightarrow$ Cys & $>250$ & +++ \\
\hline \multirow[t]{2}{*}{ KO-418 } & $1318(\mathrm{C} \rightarrow \mathrm{T})$ & 440 & $\mathrm{Arg} \rightarrow \mathrm{Cys}$ & $>250$ & ++ \\
\hline & $1280(\mathrm{~A} \rightarrow \mathrm{C})$ & 427 & $\mathrm{Asp} \rightarrow \mathrm{Ala}$ & & \\
\hline \multirow[t]{2}{*}{ KO-419 } & $1318(\mathrm{C} \rightarrow \mathrm{T})$ & 440 & Arg $\rightarrow$ Cys & $>250$ & ++ \\
\hline & $1279(\mathrm{G} \rightarrow \mathrm{A})$ & 427 & Asp $\rightarrow$ Asn & & \\
\hline KO-420 & $\begin{array}{l}\text { Reversion of } \\
1318(\mathrm{~T} \rightarrow \mathrm{C})\end{array}$ & & & 10 & - \\
\hline KO-421 & - & & & 10 & - \\
\hline KO-422 & $1309(\mathrm{C} \rightarrow \mathrm{T})$ & 437 & His $\rightarrow$ Tyr & $>250$ & ++ \\
\hline KO-423 & $1310(A \rightarrow G)$ & 437 & His $\rightarrow$ Arg & $>250$ & ++ \\
\hline
\end{tabular}

*Numbering originated from the start codon of the ORF.

†Determined after 3 days incubation on GYM agar.

$\ddagger$ Determined after 5 days incubation on R4 agar. - , No production; ++ , considerable production; +++ , abundant production. $\mathbb{S}$ Wild-type $r p o B$ gene. 
acetonitrile $/ 5 \%$ trifluoroacetic acid (TFA). The extracts were dried by using a Speed-Vac and reconstituted by adding $6 \mu \mathrm{l}$ of $50 \%$ acetonitrile $/ 0 \cdot 1 \%$ TFA. Resulting samples were spotted onto a MALDI-TOF/MS sample target with $\alpha$-cyano-4hydroxycinnamic acid (Fluka). Angiotensin II (human; Sigma) and insulin chain B (bovine; Sigma) were used for external calibration. Samples were analysed using a REFLEX II MALDI mass spectrometer (Bruker). Mascot (Matrix Science) was used to identify the protein from the mass data. For Nterminal-sequencing analysis, gel spots from the two-dimensional polyacrylamide gels were blotted onto a PVDF membrane. After staining, the spots were cut out and the membrane was subjected directly to $\mathrm{N}$-terminal-sequencing analysis using a G1000A Protein Sequencer (Hewlett Packard).

\section{RESULTS}

\section{rif mutant $\mathrm{KO}-417$ has a growth defect}

The rif mutant KO-417 (previously designated RC1) has a mutation (rif-17) that alters $\operatorname{Arg}_{440}$ to Cys in the RNA polymerase $\beta$-subunit; phenotypically, this mutant strain demonstrates the most potent ability to activate production of both the blue-coloured antibiotic Act and the red-coloured antibiotic Red (Hu et al., 2002). We first confirmed this fact using R4 medium (Fig. 1). We also found that $\mathrm{KO}-417$ has significantly impaired growth, as determined by growth of the strain in GYM liquid medium (Fig. 2). After serial transplantation of KO-417 onto GYM plates without Rif, growth-compensatory mutants were generated at a high frequency. Two types of mutants were generated. One had the ability to produce antibiotics, and mutants belonging to this type were found to have an additional $r p o B$ mutation adjacent to the original rif mutation (as represented by $\mathrm{KO}-418$ and $\mathrm{KO}-419$ ); the other lost the ability to produce antibiotics, and mutants belonging to this type were found to simply revert to the wild-type (as represented by $\mathrm{KO}-420$ ) (Table 2). The latter type no longer showed resistance to Rif. The restoration of growth was confirmed using liquid GYM medium to grow strain KO-418 (rif-17 rif-18) (Fig. 2). It should be noted that the rif double mutant KO-418 (and KO-419) still retained the ability to produce Act, although the amounts of Act produced by this strain were less than those produced by the original rif mutant, KO-417 (Fig. 1). KO-418 (and KO-419) also displayed restoration of sporulation - sporulation was severely impaired in the original strain, KO-417 (Fig. 1). The mutant strains revealed a normal ability to accumulate ppGpp, as determined by using $\mathrm{KO}-417$ and $\mathrm{KO}-418$ in a nutritional-shift-down assay (Fig. 3a).

\section{rif-17 and rif-18 are responsible for the altered phenotypes}

We next conducted gene-replacement analysis of the rif mutation. The mutant $r p o B$ alleles from KO-417 (rif-17 Act-positive) and KO-418 (rif-17 rif-18 Act-positive) were cloned into a pBluescriptSK $(+)$ vector to generate pLC1 and pLC2, respectively. Plasmids for gene replacements were constructed by inserting an apramycinresistance cassette into $\mathrm{pLC} 1$ and $\mathrm{pLC} 2$. The resulting

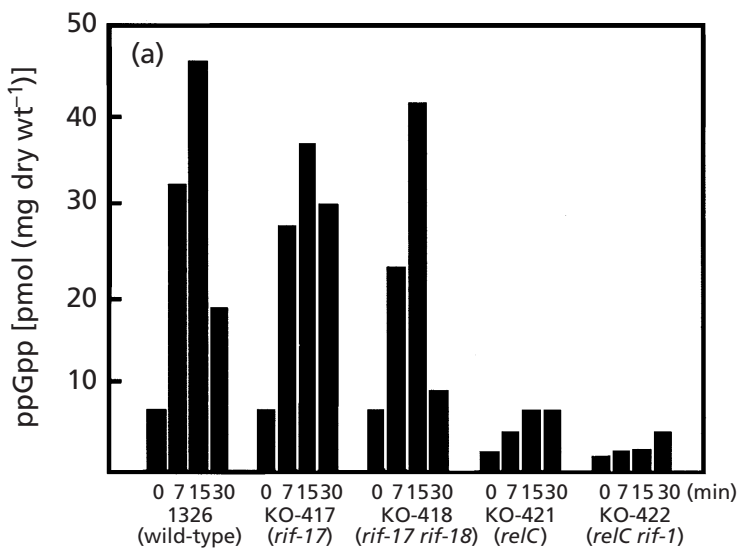

(b)

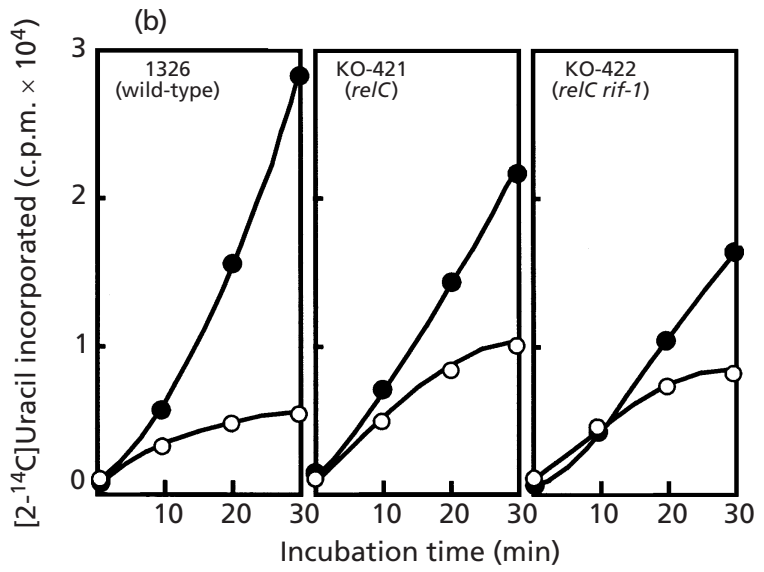

Fig. 3. Changes in (a) the intracellular concentration of ppGpp and (b) RNA synthesis after a nutritional shift-down from synthetic medium containing $2 \%$ Casamino acids to synthetic medium lacking Casamino acids. Cells from wild-type and mutant strains grown to mid-exponential phase (10-12 h incubation) in synthetic medium containing Casamino acids were harvested and transferred to fresh synthetic medium without Casamino acids and then incubated at $30^{\circ} \mathrm{C}$ with shaking. Amounts of ppGpp and RNA synthesis were determined as described in Methods. (a) ppGpp level was assayed at 0, 7, 15 and $30 \mathrm{~min}$ after the shift-down. (b) Cells, grown with Casamino acids, were collected and transferred to give an $\mathrm{OD}_{600}$ value of 2 in synthetic medium containing [2${ }^{14}$ C] uracil with $(0)$ or without $(O)$ Casamino acids and incubated for $1 \mathrm{~h}$.

plasmids, pLC3 (containing rif-17) and pLC4 (containing rif-17 rif-18), were introduced into S. lividans 1326 by protoplast transformation. Apramycin-resistant transformants were selected and passed through three rounds of non-selective cultivation on GYM agar to facilitate the second crossover. As illustrated in Fig. 4, among the apramycin-sensitive double-crossed recombinants that were derived from a single-crossed type-B recombination (apramycin-resistant, Rif-sensitive, Actnegative), we could identify two types of recombinants - type I, in which the mutant $r p o B$ gene sequence was present, and type II, which contained the wild-type $r p o B$ sequence. The ratio of type I (apramycin-sensitive, Rifresistant) to type II (apramycin-sensitive, Rif-sensitive) recombinants was $40 \%$ for pLC4, which is in agreement 
$\mathbf{A}$
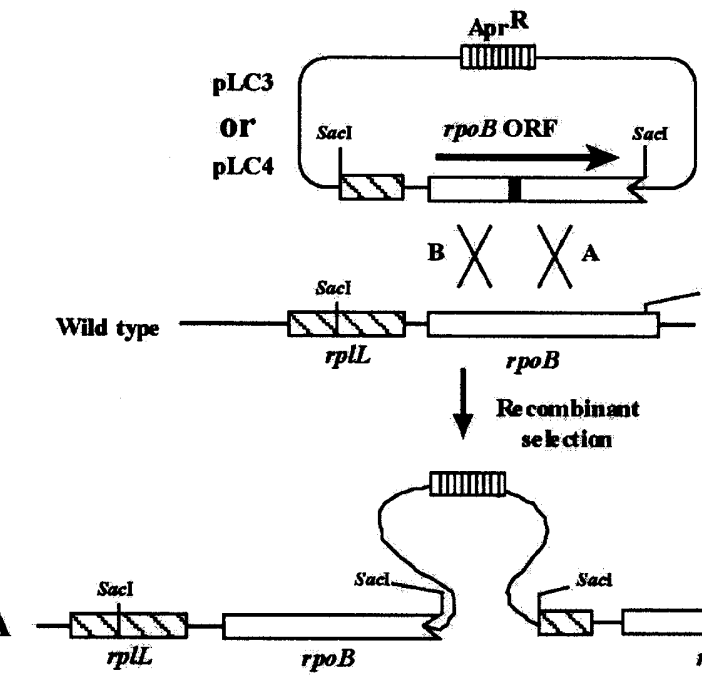

Wild type

${ }^{B} X X^{A}$

rpoB

Recombinant

selection
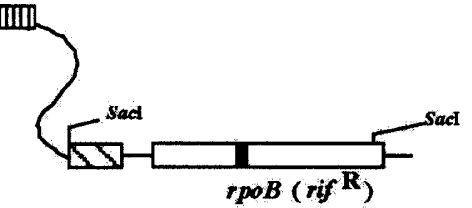

- $\frac{\text { sacl }}{r p l L}$

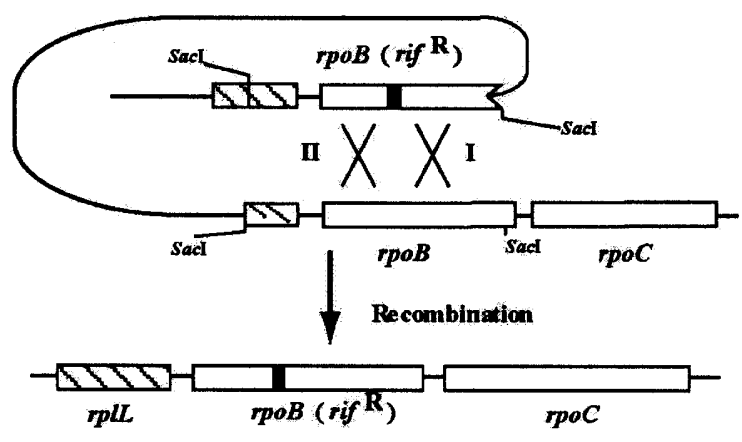

II

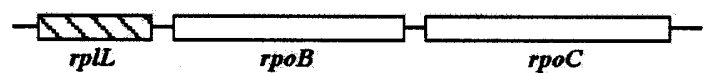

Fig. 4. Gene replacement of the $r p o B$ locus by integration of $\mathrm{pLC} 3$ or $\mathrm{pLC4}$, which contained single- or double-mutated $r p o B$ alleles. rif $^{\mathrm{R}}$ indicates the mutated $r p o B$ gene. The solid bar in the middle of $r p o B$ represents the mutation site.

with the length of the homologous fragment downstream or upstream of the rif mutation points, while the ratio of type I to type II recombinants was as low as $8 \%$ for $\mathrm{pLC} 3$, which was apparently due to the hampered growth of the rif-17 recombinants. In both cases, the Rif-resistant recombinants exhibited the Act-positive phenotype, while Rif-sensitive recombinants exhibited the Act-negative phenotype. The allele replacements were confirmed by DNA-sequence analysis (data not shown). The Rif-resistant recombinants, which were generated using pLC4 (containing rif-17 rif-18), grew as well as the wild-type strain, while the Rif-resistant recombinants, which were generated using pLC3 (containing rif-17), all grew slowly. Thus, we conclude that the mutant rpoB allele (rif-17) is responsible for Rif resistance, the observed growth defect and for activation of Act production in S. lividans, while the rif-18 mutation is responsible for the observed restoration of growth.

\section{rif effect on re/C mutation}

For S. coelicolor (and for other Streptomyces spp.), the introduction of a certain relC $(=r p l K)$ mutation into the organism severely restricts its ability to produce Act and Red, due to its failure to synthesize ppGpp (Ochi, 1990a, b; Kawamoto et al., 1997). The S. lividans wild-type strain 1326 is able to accumulate ppGpp upon nutritional shift-down (Ochi, 1989). To determine the efficacy of the rif mutation in the relC background, we screened a relC-type mutant from $S$. lividans 1326 , as described in Methods. Among the 27 thiostreptonresistant mutants that developed spontaneously, we found a possible relC mutant which had a 6 bp deletion in $r p l K$, which resulted in the loss of the dipeptide $\mathrm{Val}_{27} \mathrm{Gly}_{28}$ in the ribosomal protein L11. This mutation is of the same type as that detected in the S. coelicolor relC mutant KO-100 (Ochi et al., 1997). As expected, the presumptive relC mutant (designated KO-421) displayed 


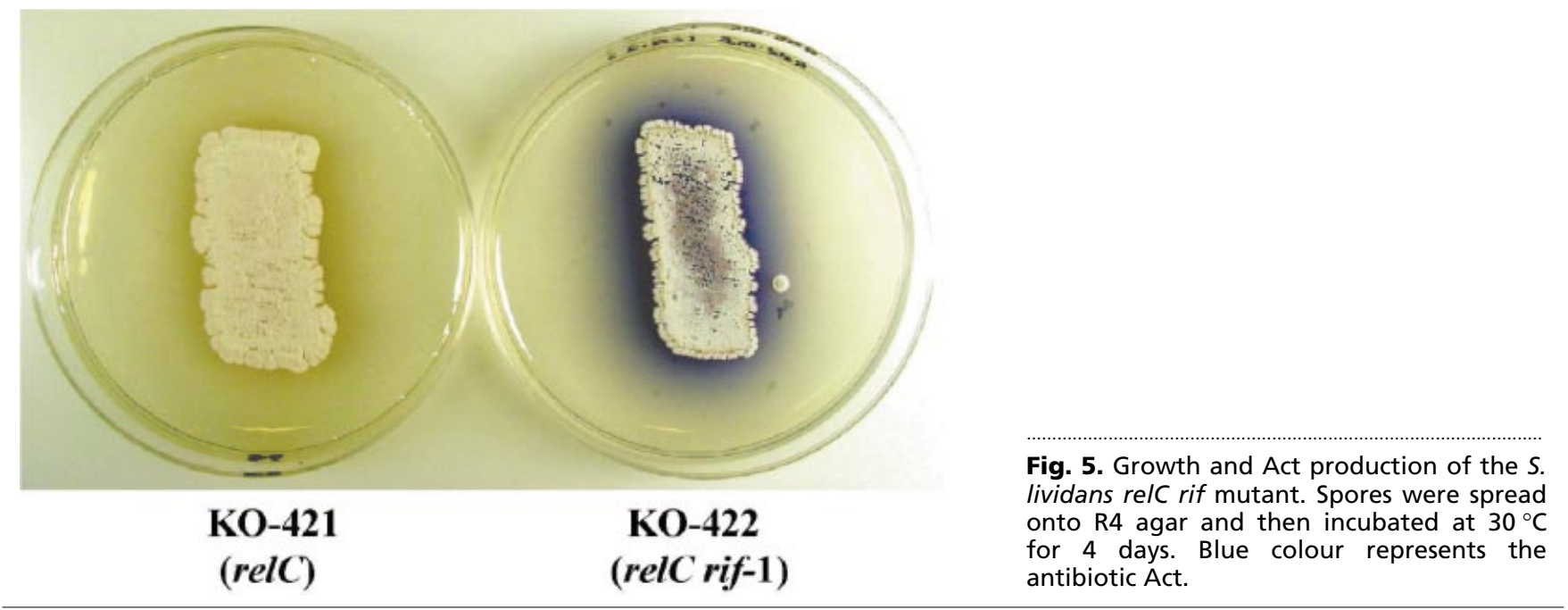

a significantly reduced ability to accumulate ppGpp as compared to the wild-type strain (Fig. 3a), and showed the relaxed response with respect to RNA synthesis upon nutritional shift-down (Fig. 3b). Thus, the thiostrepton-resistant mutant $\mathrm{KO}-421$ is a typical relC mutant.

Strikingly, among the rif mutants that were generated spontaneously from the relC strain KO-421, the Actpositive phenotype was detected at a frequency as high as $80 \%$ (81 out of 101). Two representative mutants, $\mathrm{KO}-422$ (relC rif-1) and $\mathrm{KO}-423$ (relC rif-2), can be found in Table 1 . Both mutants possess a point mutation within $r p o B$, as detected by DNA sequencing (Table 2). Act production by the relC rif double mutant KO-422 is shown in Fig. 5, as an example. These results, together with the results from the ppGpp assay, indicate that the rif effect on antibiotic production activation can be provoked even in the relC genetic background.

\section{Expression analysis of actII-ORF4}

Stationary-phase production of Act by $S$. coelicolor A3(2) is transcriptionally regulated (Takano et al., 1992; Gramajo et al., 1993). The ActII-ORF4 protein, which is encoded by the actII-ORF4 gene, has been characterized as a DNA-binding protein that positively regulates the transcription of the Act biosynthesis gene in $S$. coelicolor A3(2). We analysed the expression pattern of actII-ORF4 by Western blotting and by RTPCR, comparing the mutants with the wild-type strain. The expression level of actII-ORF4 in the S. lividans wild-type strain 1326 was threefold lower than that seen in S. coelicolor A3(2), but increased about fourfold by introducing the rif mutation rif-17 into strain 1326, in agreement with work by $\mathrm{Hu}$ et al. (2002). In contrast, expression of actII-ORF4 was found to be severely suppressed in the relC strain KO-421 (data not shown). However, introduction of the rif mutation into the relC strain provoked expression of actII-ORF4 to the level of the rif mutant KO-417, both on the transcription and translation levels (data not shown), thus accounting for the obvious production of Act in the relC rif double mutant.

\section{Effect of Casamino acid deprivation on RNA synthesis}

The rel mutants, including relA and $\mathrm{relC}$, are characterized by the relaxed response (i.e. failure to inhibit stable RNA synthesis) to amino-acid limitation (Ochi, $1990 \mathrm{a}, \mathrm{b})$. We hypothesized that the rif mutations that conferred the Act-positive phenotype may behave like 'stringent' RNA polymerases during growth in nutritionally rich media. To assess this possibility, we analysed the rate of RNA synthesis during growth in a nutritionally rich medium (i.e. synthetic medium supplemented with $2 \%$ Casamino acids), using the rif mutants KO-417 and KO-418 (Fig. 6a). These strains were grown to mid-exponential phase $[100 \mathrm{mg}$ dry cell wt $\left.(100 \mathrm{ml} \text { culture })^{-1}\right]$, and then $\left[2-{ }^{14} \mathrm{C}\right]$ uracil was added to the culture, followed by a further $60 \mathrm{~min}$ incubation. Strikingly, rif mutant KO-417 revealed a fourfold reduction in RNA synthesis, when compared to the wild-type strain. The rif double mutant KO-418 exhibited a less-pronounced reduction in RNA synthesis, but the reduction was still significant (Fig. 6a) despite having no discernible effect on growth (see Fig. 2). Likewise, the relC rif double mutant KO-422 exhibited significantly reduced RNA synthesis compared to the parental relC strain KO-422 (Fig. 6b), although the mutant also showed the relaxed response upon nutritional shift-down (Fig. 3b), reflecting the inability of the mutant to accumulate normal levels of ppGpp (Fig. 3a). Thus, the rif mutants exhibiting the Act-positive phenotype have a RNA polymerase with reduced activity for RNA synthesis during their growth in a nutritionally rich medium.

\section{Total-protein analysis in mutant and wild-type strains}

We next searched for proteins that were responsible for the remarkable differences between the rif mutants and the wild-type strain. We employed the rif double mutant 


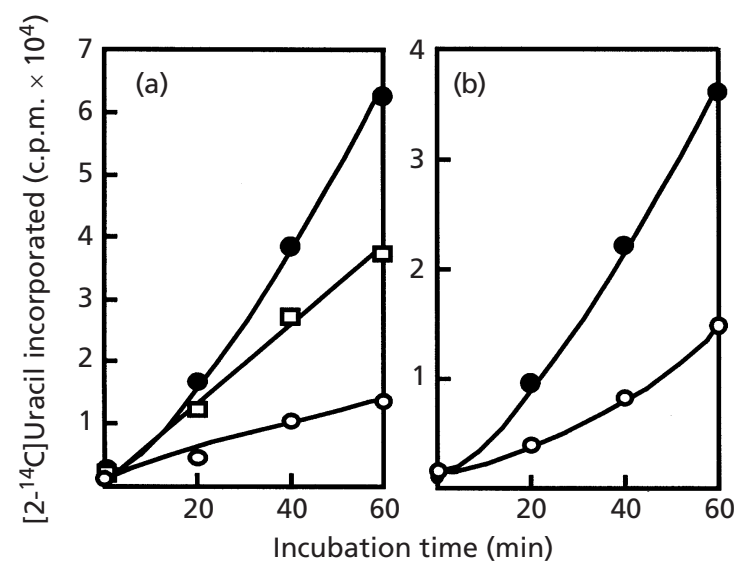

Fig. 6. RNA synthesis by the S. lividans parental strain and the rif mutants (a) or the re/C strain and the re/C rif mutant (b) during growth in synthetic medium plus $2 \%$ Casamino acids. Strains were grown to mid-exponential phase (see legend to Fig. 3) to yield $100 \mathrm{mg}$ dry cell wt $(100 \mathrm{ml} \text { culture })^{-1}$. [2${ }^{14} \mathrm{C}$ ]uracil $(0.2 \mu \mathrm{Ci}, 7.4 \mathrm{kBq})$ was added to $10 \mathrm{ml}$ aliquots of the culture to give a final concentration of $100 \mu \mathrm{M}$, and incubation was continued for $1 \mathrm{~h}$. At the indicated time, $0.5 \mathrm{ml}$ of culture was removed to measure the trichloroacetic acid-precipitable radioactivity. (a) $\bullet$, Wild-type strain 1326; O, KO-417 (rif-17); $\square$, KO-418 (rif-17 rif-18). (b) ๑, KO-421 (relC); O, KO-422 (relC rif-1).

KO-418 (rif-17 rif-18), because this mutant showed almost no impairment of growth. Strains were grown on GYM agar plates to the early $(24 \mathrm{~h})$, transition ( $36 \mathrm{~h})$ or late $(60 \mathrm{~h})$ phases of growth. After the extraction of total proteins, the protein samples were analysed by twodimensional PAGE. Eventually, we found several protein spots whose amounts differed substantially between the mutant and the wild-type strain. The difference between the spot patterns of the strains was especially pronounced in the cells grown to the transition phase (36 h), as shown in Fig. 7 (protein spots are designated by arrows). The two highlighted protein spots (A and B) were abundant in the rif mutant strain, but not in the wild-type strain. Peptide-mass-fingerprinting analysis and N-terminal-sequencing analysis, using a protein sequencer, both revealed that spot A represents glutamine synthetase (GenBank no. CAB43949), encoded by the glnII gene, and spot $\mathrm{B}$ represents oxidoreductase (GenBank no. CAC37883), encoded by the SC1G7.08c gene. The burst of expression of these two proteins in the rif mutant was temporal, since no (or at most faint) spots were detectable in cells in the early or late phase of growth (data not shown).

\section{DISCUSSION}

We have previously reported that antibiotic production by $S$. coelicolor is dramatically activated by introducing certain mutations into $r p o B$ that confer resistance to Rif (Hu \& Ochi, 2001; Hu et al., 2002). Our principal findings in this study were that (i) the expression level of actII-ORF4 increases significantly by introducing certain rif mutations into $S$. lividans, irrespective of a relpositive or relC genetic background, which is accompanied by activation of Act production and remarkable expression of oxidoreductase and glutamine synthetase, and (ii) mutant RNA polymerases behave like 'stringent' RNA polymerases. Although the wild-type strain of $S$. lividans does not produce Act, the relC mutation resulted in a significant reduction in actII-ORF4 expression. Therefore, we concluded that the dependence of S. lividans on ppGpp to express the key gene actIIORF4 (and thus for Act production) can apparently be bypassed by a certain mutation in the RNA polymerase. The low level of ActII-ORF4 protein expressed in the wild-type strain of S. lividans apparently was not enough to activate Act biosynthesis, as compared with its expression in S. coelicolor (see Results).

The present study provides evidence for the significance of the growth rate of $S$. lividans in antibiotic produc-
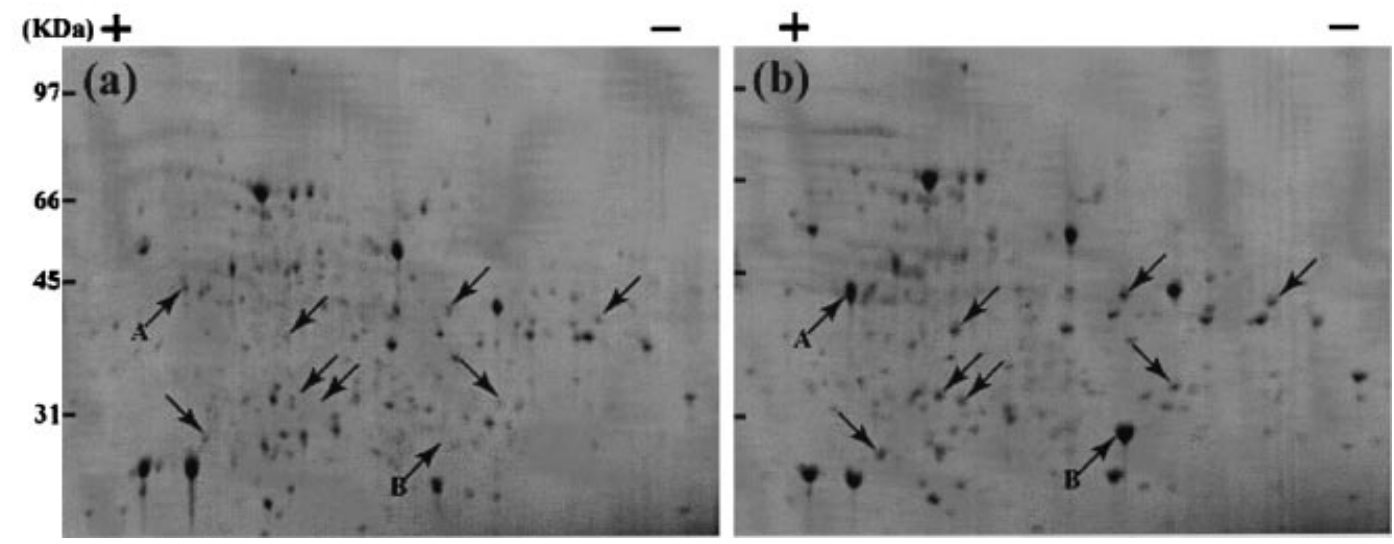

Fig. 7. Two-dimensional PAGE analysis of total proteins from (a) wild-type S. lividans and (b) mutant KO-418 (rif-17 rif-18) grown to transition $(36 \mathrm{~h})$ phase. The arrows indicate proteins that were abundant in the mutant but absent or poor in the wild-type strain, 1326. The gels were stained with Coomassie blue R250. Spots A and B were subjected to analysis by mass and by protein sequencing. Molecular mass markers are shown at the left-hand side of the images. 
tion; the growth rate of the organism was closely linked to the rate of RNA synthesis, as seen in the order wildtype strain $>$ double rif mutant $(\mathrm{KO}-418)>$ single rif mutant (KO-417). It is therefore likely that both the timing and the extent of antibiotic production by Streptomyces spp. are crucially decided by the physiological status of the RNA polymerase within the cell. ppGpp, a mediator for the stringent response, has been demonstrated to directly bind to the RNA polymerase $\beta$-subunit in E. coli (Chatterji et al., 1998). Reddy et al. (1995) also provided evidence for the location of the ppGpp-binding site on the E. coli RNA polymerase and the proximity relationship with the rif-binding domain. Detailed mapping of the promoter recognition domain on the $\beta$ subunit has been done using a collection of various RNA polymerase $\beta$-subunit mutants, each with a single amino-acid substitution. Thus, the domains for Rif sensitivity, ppGpp sensitivity, promoter selectivity and $\sigma$ assembly were found to be lined up along the $r p o B$ gene, which encodes the RNA polymerase $\beta$-subunit (Ishihama, 1988). From the result obtained by X-ray analysis of the core RNA polymerase (Zhang et al., 1999), Toulokhonov et al. (2001) proposed that the binding of ppGpp is allosteric and that the binding site is modular. Previous reports, in which various bacteria have been studied, have demonstrated that mutations in $r p o B$ are responsible for the acquisition of resistance to Rif (Aboshkiwa et al., 1995; Jin \& Gross, 1988; Singer et al., 1993). Results recently obtained in $E$. coli show that RNA polymerase mutants selected to confer prototrophy to a $\Delta r e l A \Delta s p o T$ strain can mimic the effect of ppGpp on the wild-type RNA polymerase (Barker et al., 2001). Therefore, it is reasonable to consider that the RNA polymerase with a rif-type $\beta$ subunit may be structurally similar to an RNA polymerase that has been modified by ppGpp, because numerous genetic analyses revealed that rif mutations frequently circumvent the $\mathrm{ppGpp}^{0}$ phenotype. Indeed, as demonstrated in E. coli, the mutant RNA polymerase may have altered promoter selectivity (Ishihama et al., 1990). In particular, the ppGpp-independent 'stringent RNA polymerases' have been described and the model for linking the dual aspects of the stringent response has been proposed (Zhou \& Jin, 1998). It is conceivable that the altered conformational status of the RNA polymerase resulting from rif-17 in S. lividans gave rise to different promoter selectivity (or affinity), directly or indirectly leading to the increased actII-ORF4 expression. Although the RNA polymerase with the rif-17 mutation behaved like a stringent RNA polymerase with respect to RNA synthesis (Fig. 6), we can not rule out the possibility that the mutant RNA polymerase generated different promoter selectivity that was capable of activating different pathways for the activation of antibiotic biosynthesis and, hence, did not behave as a stringent RNA polymerase. For instance, the clear difference in certain gene expression (see below) can be originated by a stringent RNA polymerase or simply by a modified promoter selectivity of the mutant RNA polymerase. Although the rif-17 mutation resulted in the abrogation of growth, which was apparently due to severe suppression of RNA synthesis (Fig. 6a), rif-18 (just adjacent to rif-17) could almost completely restore growth (Fig. 2). The effect of rif-18 on the $\beta$-subunit can also be explained by the subsequent alteration of the three-dimensional structure of this subunit.

The increase in the production of glutamine synthetase (type II) and oxidoreductase by introducing a rif mutation (Fig. 7) into S. lividans was dramatic. Glutamine synthetase, responsible for the synthesis of glutamine from $\mathrm{NH}_{4}^{+}$and glutamate, is a key enzyme in $\mathrm{NH}_{4}^{+}$assimilation and is regulated by nitrogen availability in micro-organisms, including Streptomyces strains (Fisher, 1999). At least two types of glutamine synthetase exist in bacteria, GSI (encoded by $g \ln A$ ) and GSII (encoded by glnII). Enteric bacteria and Bacillus subtilis only possess the GSI type, but Streptomyces strains are known to possess the eukaryotic-type glutamine synthetase GSII as well as GSI (Weisschuh et al., 2000). The role of the GSII enzyme in nitrogen metabolism in Streptomyces spp. is unclear. In nitrogen-fixing bacteria, GSII is preferentially expressed during nitrogen-limited growth and nitrogen fixation (Fisher, 1992). There are many kinds of oxidoreductases in S. coelicolor, as assigned by the $S$. coelicolor genome-sequencing project (http://www.sanger.ac.uk/Projects/S _coelicolor). The oxidoreductase (GenBank no. CAC37883) that was highlighted in this study is encoded by the $S C 1 G 7.08 \mathrm{c}$ gene, which is located near the type I polyketide synthesis gene cluster. Although our results implicate the intrinsic role of glutamine synthetase and oxidoreductase in secondary metabolism in S. lividans, further investigations are required to establish a causal relationship between these two enzymes and secondary metabolism.

\section{ACKNOWLEDGEMENTS}

This work was supported by a grant from the Organized Research Combination Systems (ORCS) of the Science and Technology Agency of Japan. We are grateful to A. Lezhava and Y. Jin for their valuable suggestions in the proteomic analysis.

\section{REFERENCES}

Aboshkiwa, M., Rowland, G. \& Coleman, G. (1995). Nucleotide sequence of the Staphylococcus aureus RNA polymerase $r p o B$ gene and comparison of its predicted amino acid sequence with those of other bacteria. Biochim Biophys Acta 1262, 73-78.

Barker, M. M., Gaal, T. \& Gourse, R. L. (2001). Mechanism of regulation of transcription initiation by ppGpp. II. Models for positive control based on properties of RNAP mutants and competition for RNAP. J Mol Biol 305, 689-702.

Bibb, M. J. (1996). 1995 Colworth Prize Lecture. The regulation of antibiotic production in Streptomyces coelicolor A3(2). Microbiology 142, 1335-1344.

Champness, W. C. \& Chater, K. F. (1994). Regulation and integration of antibiotic production and morphological differentiation in Streptomyces spp. In Regulation of Bacterial Differentiation, pp. 61-93. Edited by P. Piggot, C. P. Moran \& P. Youngman. Washington, DC: American Society for Microbiology. 
Chater, K. F. (1993). Genetics of differentiation in Streptomyces. Annu Rev Microbiol 47, 685-713.

Chatterji, D., Fujita, N. \& Ishihama, A. (1998). The mediator for stringent control, ppGpp, binds to the $\beta$-subunit of Escherichia coli RNA polymerase. Genes Cells 3, 279-287.

Demain, A. L. \& Fang, A. (1995). Emerging concepts of secondary metabolism in actinomycetes. Actinomycetologica 9, 98-117.

Fisher, S. H. (1992). Glutamine synthesis in Streptomyces-a review. Gene 115, 13-17.

Fisher, S. H. (1999). Regulation of nitrogen metabolism in Bacillus subtilis: vive la difference! Mol Microbiol 32, 223-232.

Gramajo, H. C., Takano, E. \& Bibb, M. J. (1993). Stationary-phase production of the antibiotic actinorhodin in Streptomyces coelicolor A3(2) is transcriptionally regulated. Mol Microbiol 7, 837-845.

Hosoya, Y., Okamoto, S., Muramatsu, H. \& Ochi, K. (1998). Acquisition of certain streptomycin-resistant $(s t r)$ mutations enhances antibiotic production in bacteria. Antimicrob Agents Chemother 42, 2041-2047.

Hu, H. \& Ochi, K. (2001). Novel approach for improving the productivity of antibiotic-producing strains by inducing combined resistant mutations. Appl Environ Microbiol 67, 1885-1892.

Hu, H., Zhang, Q. \& Ochi, K. (2002). Activation of antibiotic biosynthesis by specified mutations in the $r p o B$ gene (encoding RNA polymerase $\beta$ subunit) of Streptomyces lividans. J Bacteriol 184, 3984-3991.

Ishihama, A. (1988). Promoter selectivity of prokaryotic RNA polymerase. Trends Genet 4, 282-286.

Ishihama, A., Fujita, N., Igarashi, K. \& Ueshima, R. (1990). Structural and functional modulation of Escherichia coli RNA polymerase. In Structure and Function of Nucleic Acids and Proteins, pp. 153-159. Edited by Y. Felicia, H. Wu \& W. W. Cheng. New York: Raven.

Jin, D. J. \& Gross, C. A. (1988). Mapping and sequencing of mutations in the Escherichia coli rpoB gene that lead to rifampicin resistance. J Mol Biol 202, 45-58.

Kawamoto, S., Zhang, D. \& Ochi, K. (1997). Molecular analysis of the ribosomal L11 protein gene $(r p l K=$ relC) of Streptomyces griseus and identification of a deletion allele. Mol Gen Genet 255, 549-560.

Kieser, T., Bibb, M. J., Butter, M. J., Chater, K. F. \& Hopwood, D. A. (2000). Practical Streptomyces Genetics. Norwich: John Innes Foundation.

Leblond, P., Redenbach, M. \& Cullum, J. (1993). Physical map of the Streptomyces lividans 66 genome and comparison with that of the related strain Streptomyces coelicolor A3(2). J Bacteriol 175, 3422-3429.

Martin, J. F. \& Liras, P. (1989). Organization and expression of genes involved in the biosynthesis of antibiotics and other secondary metabolites. Annu Rev Microbiol 43, 173-206.

Ochi, K. (1987). Metabolic initiation of differentiation and secondary metabolism by Streptomyces griseus: significance of the stringent response (ppGpp) and GTP content in relation to A factor. J Bacteriol 169, 3608-3616.
Ochi, K. (1989). The tsr gene-coding plasmid pIJ702 prevents thiopeptin from inhibiting ppGpp synthesis in Streptomyces lividans. FEMS Microbiol Lett 61, 219-224.

Ochi, K. (1990a). A relaxed (rel) mutant of Streptomyces coelicolor A3(2) with a missing ribosomal protein lacks the ability to accumulate ppGpp, A-factor and prodigiosin. J Gen Microbiol 136, 2405-2412.

Ochi, K. (1990b). Streptomyces relC mutants with an altered ribosomal protein ST-L11 and genetic analysis of a Streptomyces griseus relC mutant. J Bacteriol 172, 4008-4016.

Ochi, K., Zhang, D., Kawamoto, S. \& Hesketh, A. (1997). Molecular and functional analysis of the ribosomal L11 and S12 protein genes ( $r p l K$ and $r p s L)$ of Streptomyces coelicolor A3(2). Mol Gen Genet 256, 488-498.

Reddy, P. S., Raghavan, A. \& Chatterji, D. (1995). Evidence for a ppGpp-binding site on Escherichia coli RNA polymerase: proximity relationship with the rifampicin-binding domain. Mol Microbiol 15, 255-265.

Redenbach, M., Kieser, H. M., Denapaite, D., Eichner, A., Cullum, J., Kinashi, H. and Hopwood, D. A. (1996). A set of ordered cosmids and a detailed genetic and physical map for the $8 \mathrm{Mb}$ Streptomyces coelicolor A3(2) chromosome. Mol Microbiol 21, 77-96.

Sambrook, J., Fritsch, E. F. \& Maniatis, T. (1989). Molecular Cloning: a Laboratory Manual, 2nd edn. Cold Spring Harbor, NY : Cold Spring Harbor Laboratory.

Shima, J., Hesketh, A., Okamoto, S., Kawamoto, S. \& Ochi, K. (1996). Induction of actinorhodin production by rpsL (encoding ribosomal protein S12) mutations that confer streptomycin resistance in Streptomyces lividans and Streptomyces coelicolor A3(2). J Bacteriol 178, 7276-7284.

Singer, M., Jin, D. J., Walter, W. A. \& Gross, C. A. (1993). Genetic evidence for the interaction between cluster I and cluster III rifampicin resistant mutations. J Mol Biol 231, 1-5.

Takano, E., Gramajo, H. C., Strauch, E., Andres, N., White, J. \& Bibb, M. J. (1992). Transcriptional regulation of the redD transcriptional activator gene accounts for growth-phase-dependent production of the antibiotic undecylprodigiosin in Streptomyces coelicolor A3(2). Mol Microbiol 6, 2797-2804.

Toulokhonov, I. I., Shulgina, I. \& Hernandez, V. J. (2001). Binding of the transcription effector ppGpp to Escherichia coli RNA polymerase is allosteric, modular, and occurs near the $\mathrm{N}$ terminus of the $\beta^{\prime}$-subunit. J Biol Chem 276, 1220-1225.

Weisschuh, N., Fink, D., Vierling, S., Bibb, M. J., Wohlleben, W. \& Engels, A. (2000). Transcriptional analysis of the gene for glutamine synthetase II and two upstream genes in Streptomyces coelicolor A3(2). Mol Gen Genet 264, 461-469.

Zhang, G., Campbell, E. A., Minakhin, L., Richter, C., Severinov, K. \& Darst, S. A. (1999). Crystal structure of Thermus aquaticus core RNA polymerase at $3 \cdot 3 \AA$ resolution. Cell $98,811-824$.

Zhou, Y. N. \& Jin, D. J. (1998). The $r p o B$ mutants destabilizing initiation complexes at stringently controlled promoters behave like 'stringent' RNA polymerases in Escherichia coli. Proc Natl Acad Sci U S A 95, 2908-2913.

Received 30 April 2002; revised 10 July 2002; accepted 20 August 2002. 